\title{
The Dark Box instrument for fast automatic testing of the photomultipliers for KM3NeT
}

\author{
Carlos Maximiliano Mollo \\ INFN-Napoli \\ Complesso Universitario di Monte Sant'Angelo via Cintia ed. 6 - 80126 Napoli,Italy \\ E-mail: maximilena.infn.it
}

Paolo Piattelli ${ }^{1}$

LNS - INFN - Catania

Via S. Sofia 62 - 95123 Catania,Italy

E-mail: piattellialns.infn.it

on behalf of the KM3NeT Collaboration

Since the early days of experimental particles physics photomultipliers have played an important role in detector design. Also in astroparticle physics research, photomultipliers are largely used, in particular in experiments employing the technique of the detection of Cherenkov photons. Currently, the KM3NeT Collaboration [1] is building a water Cherenkov neutrino telescope in the Mediterranean Sea based on the next generation optical modules with multiple low price 3-inch photomultiplier tubes. In its final layout, the KM3NeT neutrino telescope will host several hundred thousand photomultipliers, which must be tested and calibrated during the production of the optical modules. To overcome a possible bottleneck in the production process of testing and calibration of the massive amount of photomultipliers for KM3NeT, we developed the Dark Box instrument to accelerate the process.

The Dark Box setup is designed to provide fast simultaneous automatic testing of 62 photomultipliers to verify their compliance to requirements for timing and ToT resolution and the occurrence of spurious pulses. In addition, the Dark Box can be easily converted into a general instrument for testing and calibrating large amounts of photomultipliers other than those for KM3NeT.

We report on the design and performance of the Dark Box instrument for the high-statistics measurement of the characteristics of photomultipliers and of their calibration.

The 34th International Cosmic Ray Conference

30 July- 6 August, 2015

The Hague, The Netherlands 


\section{Introduction}

The KM3NeT Observatory is a large-scale neutrino telescope to be built in the deep waters of the Mediterranean Sea. With several cubic kilometers instrumented with thousands of optical sensors, KM3NeT will be the largest and most sensitive high-energy neutrino detector. It will be capable of neutrino astronomy with unprecedented accuracy. Being situated in the Northern Hemisphere it will be particularly suited to the investigation of high-energy neutrinos from our Galaxy.

The neutrino detection is based on sampling the Cherenkov light induced by the particles produced in a neutrino interaction in the vicinity of the detector. The muon produced in the charged current interaction of a muon neutrino provides through its long range in water a particularly sensitive detection channel.

The sampling of Cherenkov light in the KM3NeT telescope is performed with the Digital Optical Modules (DOMs). A prototype of these is the subject of this paper. It has been installed on the instrumentation line of the ANTARES detector.

The DOM consists of a glass sphere, $432 \mathrm{~mm}$ in diameter. The sphere houses 31 PMTs with photocathode diameter of $72 \mathrm{~mm}$. A cone-shaped reflector that effectively increases the diameter to about $85 \mathrm{~mm}$ surrounds each tube. The $31 \mathrm{PMts}$ are read simultaneously by a Central Logic board (CLB) that also ensure data reception/transmission from/to shore. A technical drawing of the DOM is shown in figure 1.

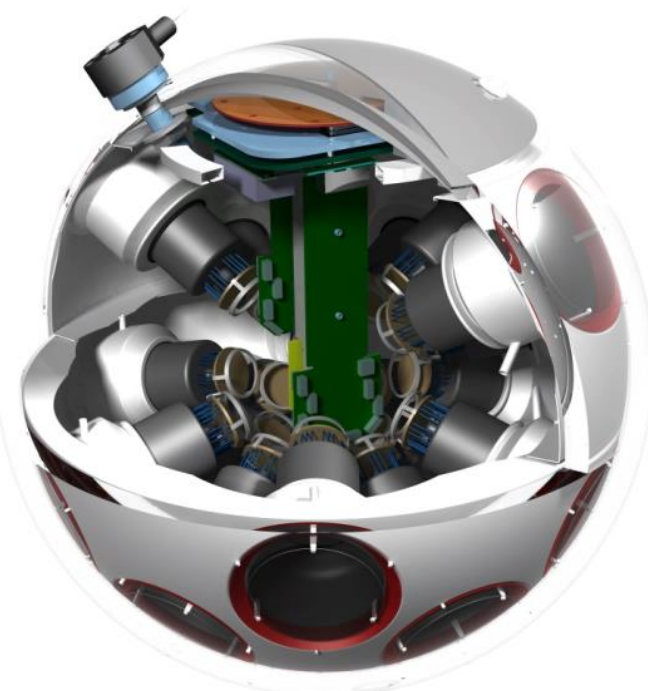

Figure 1: The DOM assembly.

The choice of 3-inch PMTs is driven by their quantum efficiency above $30 \%$, by a small transit time spread and by the fact that do not require shielding from the Earth's magnetic field. Also the reliability of the multi-PMT DOM is higher since a failure of a single PMT will have much less impact on the performance of the total DOM as compared to an optical module housing a single large PMT.

Each PMT in the DOM is equipped with an active base called "BaseBoard" that provides, thanks to a Cockcroft-Walton generator, the needed high voltage to each dynode. In addition, in the BaseBoard it is present a discriminator that converts the analog signal from the anode of the PMT into a time over threshold signal. The high voltage and other settings, like the threshold level of the discriminator, are programmed using an $\mathrm{I}^{2} \mathrm{C}$ protocol.

The drawback of this choice is the large amount of PMTs to be tested and calibrated. For the whole infrastructure we do expect more than hundred thousand PMTs. To overcome the possible bottleneck determined by the massive test and calibration of all PMTs, we developed an instrument (from now on dubbed Dark Box) capable to check if the PMTs satisfy the requirements on the timing resolution and ToT resolution, and number of the spurious pulses. The Dark Box 
The Dark Box instrument for fast automatic testing of the photomultipliers for KM3NeT

setup is designed to provide a facility for fast automatic test of 62 PMTs simultaneously.

The results quoted in this paper have been obtained with 3-inch Hamamatsu PMTs [2], but the Dark Box is able to host any 3-inch shape photomultiplier.

\section{Instrument requirements and implementation}

\subsection{Mechanics}

The Dark Box allows the simultaneous measurement of a large number of PMTs ensuring the dark tightness. Furthermore, the mechanical design must be able to ease the loading and unloading of the photomultipliers minimizing the dead times. It is also mandatory to guarantee the uniform illumination of the photocathode. This requirement imposes limitations on the design of the PMT support structure.

The solution we adopted foresees two trays that can accommodate 31 PMTs each. Each batch of 31 PMTs is read by a CLB, similarly to the final DOM configuration. The photomultipliers are maintained in a vertical position thanks to collars in PVC and elastic bands (Fig. 2 left ). Note that the photocathode is not in contact with the structure. However, to avoid falling off of the photomultiplier, trays have tiny teethes on the lower openings that are able to hold the photomultiplier during loading and unloading operations. The trays are placed on a flatbed made of PVC with 62 dark cylinders in front of each PMT. The cylinders are needed to optically isolate the PMTs among them.

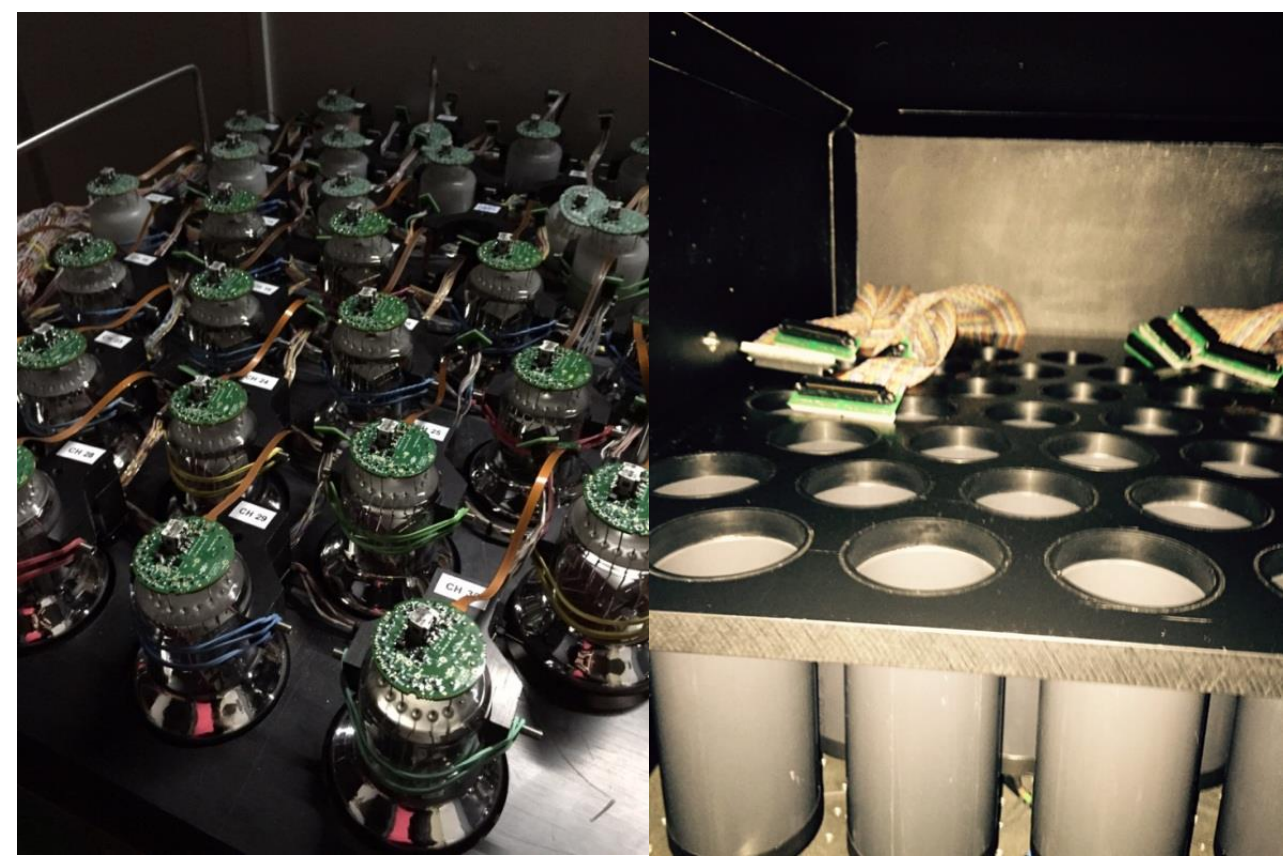

Figure 2: PMTs in the tray before the test (left). The DarkBox inner structure (right)

The dark box has been designed to be light tight. As can be seen from Figure 2 (right) particular attention has been devoted to the edges, to the corners and to the flanges for cable routing, which usually are the most critical regions to guarantee light tightness.

\section{$2.2 \quad$ Optics}

The dark box will be used to perform the following measurements on the PMTs:

- Transit Time (TT) measurements; 
The Dark Box instrument for fast automatic testing of the photomultipliers for KM3NeT

- Determination of the optimal operating voltage;

- Transit Time Spread (TTS) measurements;

- Dark count rate measurements;

- Measurements of spurious pulses (prepulses, afterpulses, delayed pulses).

The measurement of TT, TTS and spurious signals requires the illumination of the photocathode of each photomultiplier under the single photoelectron configuration. Moreover, as illustrated later while discussing the DAQ electronics, it is mandatory that the light pulses are "contemporaneous" onto each photomultiplier. Therefore, it is necessary to use a single laser source with excellent timing performance: pulse width less than $1 \mathrm{~ns}$ and jitter between the trigger from the laser controller and the light signal as small as possible, typically $<100 \mathrm{ps}$. We adopted the controller model EIG2000DX from Pilas [3] equipped with a $470 \mathrm{~nm}$ laser head. The laser is driven by the same master clock of the CLB and triggered by the PPS (Pulse per second) of the CLB. This setup ensures a jitter between the synchronization trigger output and optical signal that is typically less than 50 ps with an optical signal pulse width of about 30 ps (FWHM).

In order to illuminate all photomultipliers with a single laser source a custom optical splitter was designed and installed. Its characteristics are: good uniformity in the power distribution, same length (accuracy $<1 \mathrm{~cm}$ ) to ensure equal arrival time of the light signals onto each photomultiplier. The solution adopted is shown in figure 3 where the working principle of the splitter is shown. It is an optical splitter 1 to 70 where the 70 output fibers have a core of $100 \mu \mathrm{m}$ and are illuminated with an input fiber having a core diameter of $600 \mu \mathrm{m}$. The output power distribution for each channel of the splitter is shown in figure 4 . The uniformity is very good, indeed all outputs have a fraction of the input power in the range $0.5 \%-1.5 \%$.

The technique used for the realization of the splitter ensures high uniformity on the lengths of the output fibers. Consequently, the timing of the signals on each photomultiplier is guaranteed. The measurement of the TTS requires that all photocathodes be illuminated in a single photoelectron condition. Moreover, the probability that a single photon hits the photocathode should be uniform. To ensure good illumination uniformity, a diffusing opalescent disc was placed on the bottom of each cylinder. The output power from the splitter must be endlessly monitored to guarantee the single photoelectron operation mode. Therefore, an output of the splitter is connected to the probe of a power meter Newport mod. 2936-C [4](equipped with a probe Newport 918D-SL-OD3), allowing the power measurement with a sensitivity of $0.4 \mathrm{pW}$.

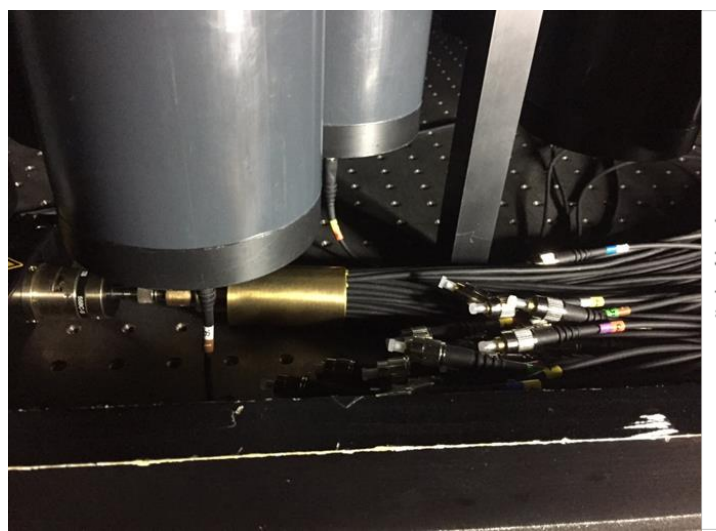

Figure 3: The optical splitter inside the Dark Box. Figure 4: The splitter output power distribution, all power output are within $0.5 \%$ and $1.5 \%$ of the input power. 
The Dark Box instrument for fast automatic testing of the photomultipliers for KM3NeT

\subsection{Electronics}

The DAQ electronics is based on the KM3NeT Central Logic Board (CLB). The CLB, is the main logic board of the KM3NeT DOM. It is directly connected with a Power Conversion Board which provides all the needed rails for the board $(1.0 \mathrm{~V}, 1.8 \mathrm{~V}, 2.5 \mathrm{~V}, 3.3 \mathrm{~V}, 5.0 \mathrm{~V})$. The purpose of the CLB is to manage all the instrumentation present inside the DOM, acquire incoming data from the 31 PMTs and from the acoustic device, pack the data into User Datagram Protocol (UDP) packets and send them through the optical line. A time synchronization of $1 \mathrm{~ns}$ level between all the DOMs is achieved by the usage of White Rabbit. The CLB is based on a Xilinx Kintex 7 FPGA, which is connected to all the rest of the board, including in particular:

- 1 Small Form-factor Pluggable (SFP) connector for the optical communication

- 2 custom Octopus connectors for the PMTs signals

- Tunable oscillators (White Rabbit compliant)

- Embedded instrumentations (temperature and humidity sensor, tilt \& compass)

- External connectors (for Nanobeacon LED, acoustic devices, expansion boards)

For the purposes of the Dark Box we only use the White Rabbit gear and acquire the data from PMTs. In order to acquire data from 62 PMTs simultaneously we used two CLBs synchronized using a White Rabbit switch. The LASER pulse emission is synchronized and synthonised to the CLB common clock and absolute time permitting measurement of PMT TT [5].

\subsection{Readout, Acquisition and Analysis Software}

The most part of the software tools were taken or re-adapted from the KM3NeT DAQ system, which was still in development phase (wiki.km3net.org). The software chain, together with providing fast analysis of the PMTs characteristics, has to be interfaced with the KM3NeT Database System. This step is necessary to: retrieve in real time information on the PMT assembly (e.g. serial number, Vendor's HV setting, test and integration history); store the results of the calibration and make them available to the DOM integration teams for cros checks and further analysis.

The software tools used for the dark room test bench are:

- Detector Manager written with Mono which is capable to communicate with all CLBs in the detector via SRP protocol.

- Java Remote Control, which is capable to communicate with one CLB per time and was used for debug purposes.

- CLBSwissKnife a program which analyses UDP packets from the CLB and capable to save the binary data inside them. Two types of the data was analyzed - monitoring data and optic data. The first one contains number of hits from each channel in the timeslice. The second contains arrival time (timestamp) and ToT of each hit.

The DarkBox control was realized as a Java application, which allows to manage several processes:

- Creates and fills a new directory with data for each new test set, in particular:

$\circ \quad$ Unique ID for each test (chosen by operator)

- Start/stop time of each test

- Raw data

- Analyzed ROOT data

○ Promis ID, PMT UPI, Vendor HV (HV0), tuned HV, XML upload file to the Data Base

- Continue test at any phase by loading its data directory

- Interfaces with Detector Manager through http requests to: 
The Dark Box instrument for fast automatic testing of the photomultipliers for KM3NeT

- Read PROMIS ID of the PMT bases

- Set HV for each PMT manually and monitor the values

- Set run number

- Start and stop the run

- Interfaces with Data Base through http requests to:

- Resolve PMT UPI from the PROMIS ID

○ Obtain Vendor HV (HV0) for each PMT

- Launch CLBSwissKnife

- Launch data analysis

- Launch XML upload file creation

The data analysis code is as a C++ program interfaced with ROOT libraries to create ROOT files and text output with the final plots and values. XML file creator is a ROOT script which creates a file with the PMT test results to be uploaded to the database. Also it generates a separate PDF test sheet for each PMT assembly with the test results.

\section{PMT Assembly Calibration procedure}

The PMT assembly calibration procedure consists of three main phases: HV tuning, darkening and PMT tests with laser.

\subsection{High Voltage tuning}

High voltage tuning is done to obtain the same value of ToT peak time for all PMTs. The ToT peak time value of $26.4 \mathrm{~ns}$ was chosen according to the tests done at Erlangen and corresponds to the average value for set of 60 PMTs with tuned $3 * 10^{6}$ gain and 0.3 p.e. threshold. The tuning itself is done at a fixed threshold of $1096 \mathrm{mV}$ (which corresponds to $\sim 0.3$ p.e.) in steps of $25 \mathrm{~V}$ in the range $[\mathrm{H} 0-100 \mathrm{~V} ; \mathrm{H} 0+50 \mathrm{~V}]$, where negative voltage value $\mathrm{H} 0$ is provided by the PMTs manufacturer. For each voltage the ToT distribution is collected (fig. 5 a) and fitted with Gauss distribution in the range of [maximum bin- $4 \mathrm{~ns}$; maximum bin $+\mathrm{ns}$ ] to obtain a ToT peak time. The obtained ToT peak time is plotted as a function of HV (7 points graph), fitted with a parabola and the HV value corresponding to $26.4 \mathrm{~ns}$ (that corresponds to a PMT gain of $10^{6}$ ) is taken from the fit (fig. $5 \mathrm{~b}$ ). This $\mathrm{HV}$ value considered to be acceptable if it falls in the range of [H0-150 V; $\mathrm{H} 0+50 \mathrm{~V}]$.
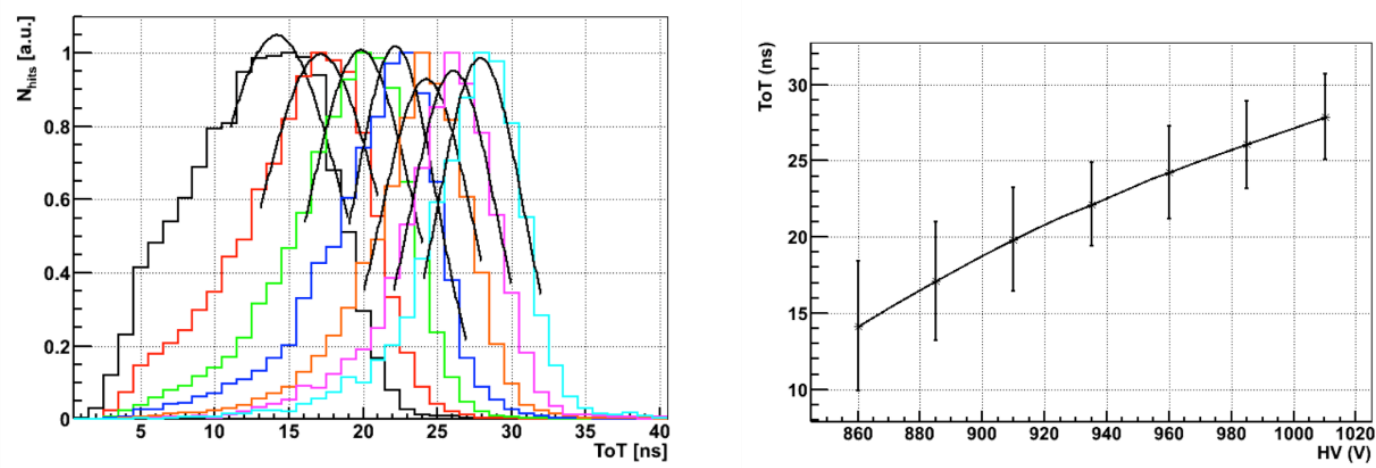

Figure 5: (a) Gaussian fit of ToT distributions from HV $=\mathrm{H} 0-100 \mathrm{~V}$ (black) to $\mathrm{H} 0+50 \mathrm{~V}$ with a step of $25 \mathrm{~V}$ and (b) parabola fit of the ToV vs HV distribution. 
The Dark Box instrument for fast automatic testing of the photomultipliers for KM3NeT

\subsection{Darkening}

The optimized HV is set to each PMT and the rates are monitored for 9 hours. The mean dark count rate is computed by an average over the last $100 \mathrm{~s}$ of the run.

\subsection{PMT tests with laser}

Two types of the hits are distinguished for the tests with laser:

- First hits: arrive in coincidence with the laser trigger in the window [T0 - $100 \mathrm{~ns}$, T0 + $100 \mathrm{~ns}$ ], where T0 is expected photons arrival time to PMT (estimated from the optical path). Also they should have no hits before them in the same time window

- After-pulses: hits which have first hit before them

Number of delayed hits estimated as a ratio of the hits in the window [main peak time $+15 \mathrm{~ns}$, main peak time $+60 \mathrm{~ns}$ ] to the number of first hits. Number of pre-pulses hits estimated as a ratio of the hits in the window [main peak time - $60 \mathrm{~ns}$, main peak time $-10 \mathrm{~ns}$ ] to the number of first hits. Number of afterpulses for type II that are due to ionization is estimated as a ratio of the afterpulses in the time window [main peak time $+60 \mathrm{~ns}$, main peak time $+10 \mu \mathrm{s}$ ]. These ratios are corrected for the dark rate hits entering the both first hits and afterpulses calculation.

ToT measurement as Gaussian fit in the window [maximum bin- $4 \mathrm{~ns}$; maximum bin $+\mathrm{ns}$ ] is done for the first hits.

\section{System performance}

The full test and calibration procedure for 62 PMTs takes about 12 hours. During the test almost all the PMT characteristics are measured. At the end of a test session all raw data and processed data are recorded into the KM3NeT Data Base in order to be available in each integration site and allow fast DOM integration. To allow an immediate check the characteristics of each PMT the software generates a PDF datasheet (fig. 6) with the most useful information and plots as: tuned HV, Dark Counts, ToT distribution, spurious pulses, TTS, etc.
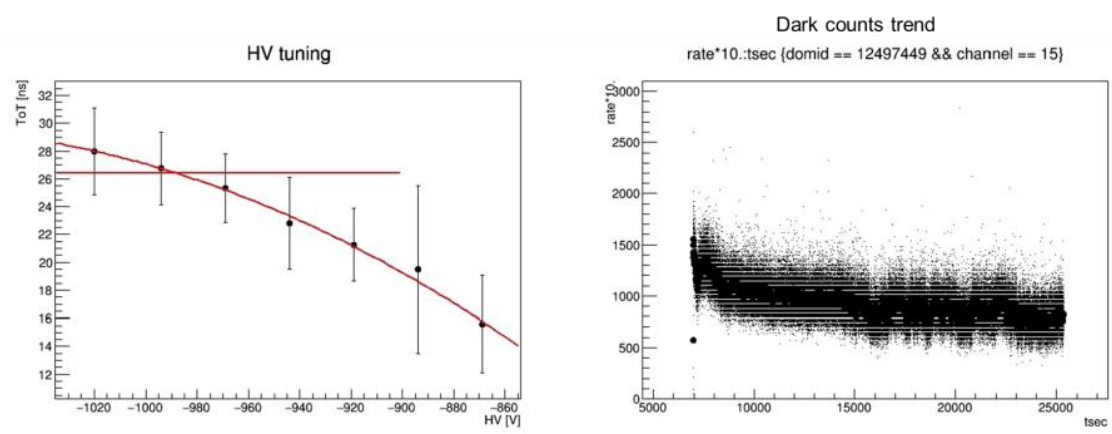

DarkBox test summary (Test \#33) PROMIS ID: $0005 \mathrm{C} 4$ PROMIS ID: 0005C4 Quality : GREEN
UPI : $3.4 .2 .3 /$ HAMA-R12199/2.4082 Tuned HV : $-987.94 \mathrm{~V}$ DarkRate : $793.13 \mathrm{~Hz}$ ToT peak : $26.8561 \mathrm{~ns}$ Prepulses : 0.00292931 Delayed : $0.0709103 \%$ Afterpulses : 0.47664 TT peak : 3793 ns
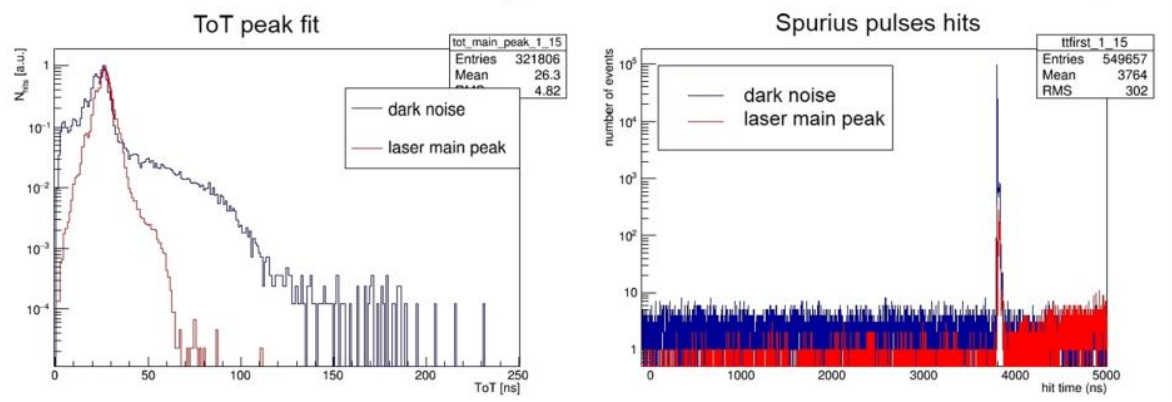

Figure 6: Automatic generated datasheet for each PMT after the test. 
The Dark Box instrument for fast automatic testing of the photomultipliers for KM3NeT

In figure 7 are shown the distributions of Dark Counts, ToT, TTS, and Tuned HV for about 600 PMTs.
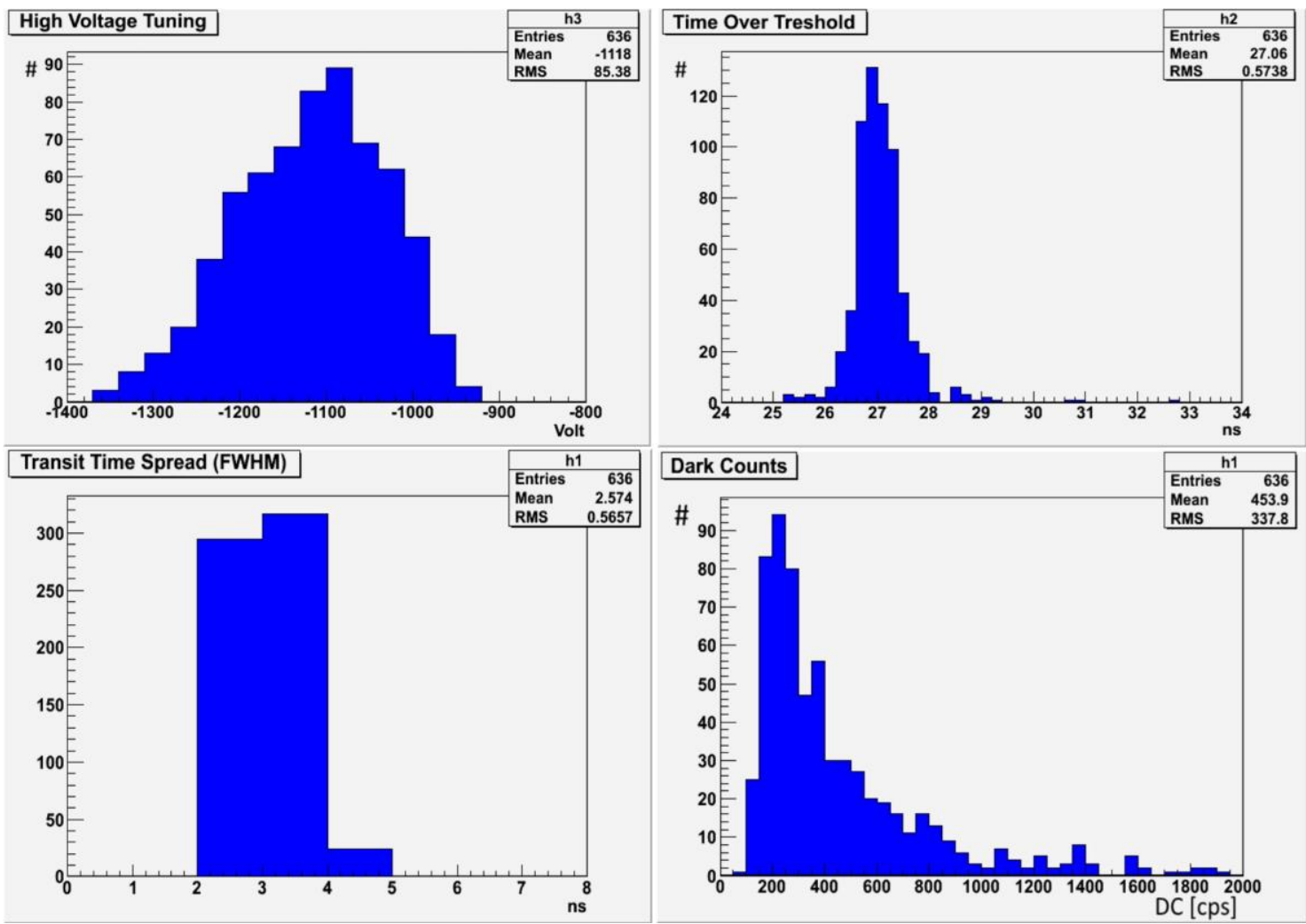

Figure 7: Distributions of the results of the test of 636 PMTs

\section{Conclusions}

The Dark Box system allows to test up to 124 PMTs per day, with usual methods the test of such number of PMTs would require weeks or months. The performances in terms of reliability were tested by comparing the test results obtained with the Dark Box with data from Hamamatsu and supported by further cross checks made at ECAP in Erlangen (Germany). To speed up the sorting of PMTs to the integration sites it was adopted a triage (RED, YELLOW, GREEN) that allows a quick selection of those PMTs ready to be integrated ("GREEN"). The PMTs that presents characteristics out of specification are labelled "RED" or "YELLOW" depending on how much deviates from the acceptable values. In conclusion, the system is in all aspects (amount of PMTs tested daily, reliability, safety, etc.) ready for the mass production of the KM3NeT neutrino telescope.

\section{References}

[1] KM3NeT collaboration - http://www.km3net.org/collaboration.php

[2] KM3NeT consortium, O. Kalekin et al. Photomultipliers for the KM3NeT optical modules. Nuclear Instruments and Methods in Physics Research A 695 (2012) 313-316

[3] LASER PiLas - http://www.alsgmbh.com/

[4] Power meter Newport - http://www.newport.com

[5]White Rabbit Switch - http://www.ohwr.org/projects/white-rabbit/wiki/Switch 